\title{
GRABADOS RUPESTRES EN EL SECTOR SEPTENTRIONAL DEL CAMPO VOLCÁNICO DE PALI AIKE, PROVINCIA DE SANTA CRUZ, ARGENTINA: VARIABILIDAD EN EL "ESTILO RÍO CHICO"
}

\author{
LILIANA M. MANZI ${ }^{\mathrm{a}}$, FLAVIA CARBALLO MARINA ${ }^{\mathrm{b}}$ \& PATRICIA A. CAMPAN
}

\begin{abstract}
RESUMEN
Se presenta el primer registro de grabados rupestres correspondientes al "estilo Río Chico" que amplía la variabilidad de técnicas de ejecución detectadas dentro del repertorio de motivos asignables a dicho estilo. Los grabados se localizan en el curso medio del río Gallegos, en el sector septentrional del Campo Volcánico de Pali Aike -CVPA- (provincia de Santa Cruz, Argentina). La coocurrencia de pinturas y grabados rupestres muy circunscripta espacialmente y vinculada a cuerpos de agua de grandes dimensiones (Laguna Potrok Aike y Bajo El Cóndor) refuerza una recurrencia en el uso humano en esos espacios con respecto al resto de la cuenca media del río Gallegos.
\end{abstract}

PALABRAS CLAVE: "Estilo Río Chico", motivos rupestres, pinturas, grabados

\section{ROCK ENGRAVINGS IN THE NORTHERN SECTION OF PALI AIKE VOLCANIC FIELD, PROVINCE OF SANTA CRUZ, ARGENTINA: VARIABILITY IN THE "STYLE RÍO CHICO".}

\begin{abstract}
The first record of rock engravings of the "Río Chico style" is presented. This record expands the variability of techniques attributable to this style.

The rock engravings are located in the middle course of the Gallegos river, in the northern sector of Pali Aike Volcanics Field -CVPA- (province of Santa Cruz, Argentina). The co-ocurrence of paintings and rock engravings in relation to large water bodies (Laguna Potrok Aike and Bajo El Cóndor) reinforces a recurrent human use of these areas in relation to the rest of the Gallegos basin.
\end{abstract}

KEY WORDS: "Río Chico style"; rock engravings motifs; art paintings; rock engravings.

a CONICET-IMHICIHU, Saavedra 15, 5 Piso, 1083 Ciudad Autónoma de Buenos Aires, Argentina. Im_manzi@yahoo.com.ar

b ICASUR-Unidad Académica Río Gallegos-UNPA., Piloto "Lero Rivera" s/n. Río Gallegos, Argentina. flaviacarballomarina@ gmail.com

c ICASUR-Unidad Académica Río Gallegos-UNPA., Piloto "Lero Rivera" s/n. Río Gallegos, Argentina. Museo Regional Padre Molina, Secretaría de Estado de Cultura de la provincia de Santa Cruz. pxcampan@gmail.com. 


\section{INTRODUCCIÓN Y MARCO REGIONAL}

Este trabajo describe y analiza el primer registro de grabados rupestres, tipo de manifestación visual que no cuenta con precedentes en la cuenca del río Gallegos. Así, se amplía la variabilidad de manifestaciones rupestres en el sector septentrional del Campo Volcánico de Pali Aike -CVPA- (provincia de Santa Cruz, Argentina). Este sector es atravesado en sentido oeste-este por la cuenca del río Gallegos y se corresponde con las expresiones volcánicas de la denominada Unidad 1, caracterizada por la presencia de coladas basálticas (sensu D'Orazio et al. 2000). Aquí, las manifestaciones rupestres más frecuentes y con más amplia distribución espacial comprenden motivos pintados y se relacionan tanto con el río Gallegos como con su tributario, el río Chico. Por sus diseños pueden ser englobadas dentro del "estilo Río Chico" definido por Bate (1970, 1971) en Última Esperanza, Chile, y posteriormente incluidos por Massone (1982) dentro del "estilo geométrico". El mencionado estilo se caracteriza por motivos geométricos simples -tales como trazos-, geométricos complejos -como puntos enmarcados- y figurativos, entre ellos zoomorfos, antropomorfos, tridígitos y manos en negativo y positivo (Bate, 1970, 1971; Campan et al. 2007; Charlin \& Borrero, 2012; Charlin, 2014; Gómez Otero, 1983-85, 1988, 1989-90; Hernández Llosas et al. 1999; Manzi et al. 2010; Manzi \& Carballo Marina, 2012; Massone, 1982; Molina, 1972; Sanguinetti de Bórmida, 1981). Las series tonales están conformadas mayoritariamente por tonos bermellón, escarlata y carmesí (sensu Maerz \& Paul, 1930) y escasos motivos negros y blancos.

Las investigaciones efectuadas en la cuenca media del río Gallegos permitieron constatar la presencia de pinturas rupestres en la margen norte del río en el Abrigo Pintado de Güer Aike -APGA- mencionado por Molina (1972), además, de registros detallados de frecuencias y clases de motivos en el Alero de Güer Aike 1 -AGA1(estancia Güer Aike) y en Campo Las Piedras -CLP(estancia Bella Vista) (Manzi et al. 2010; Manzi \& Carballo Marina, 2012) (Fig. 1).

En la margen sur del río, en la estancia Las Buitreras, se cuenta con referencias de motivos en el Abrigo de los Pescadores, la Gruta Pintada de Las Buitreras (Molina, 1972, p. 155) y en los sitios Las Buitreras Cuevas II y III -LB2 y LB3(Sanguinetti de Bórmida, 1981, p. 197). A partir de estas menciones y del reconocimiento de los sitios Meseta Las Buitreras 1 y 3 -MLB1 y MLB3se procedió a efectuar un relevamiento sistemático en el APGA, por lo que fue redenominado Meseta Las Buitreras 2 (MLB2) (Manzi \& Carballo Marina, 2012), y de las pinturas localizadas en LB2 observaciones personales- (Fig. 1). En la estancia La Carlota, en proximidad de la laguna Potrok Aike, se detectaron la Cueva 1 -PAC1- y el Alero 1 -PAA1(Gómez Otero, 1983-1985). En forma reciente, en los afloramientos volcánicos localizados al noroeste de la laguna se detectaron varias concentraciones y abrigos rocosos con pinturas rupestres en tonos de rojo, incluyendo algunos motivos en blanco. En estos conjuntos los motivos abstractos son los más frecuentes seguidos por los positivos de manos (Charlin, 2014).

Próximo al casco de esta misma estancia está emplazado el alero Romario Barría -ARB(Campan et al. 2007) y en cercanías a una cuenca endorreica llamada Bajo El Cóndor o Bajo Bella Vista, se relevaron otros dos sitios los que fueron denominados Alero de las Ortigas y Alero del Artista -AO y AA-. Mientras que, en la estancia Bella Vista fueron registradas pinturas en los sitios Puesto Cañadón Verde -PCV- y en Puesto El Cóndor -PEC- (Manzi \& Carballo Marina, 2012) (Fig. 1).

En relación con el río Chico, en la estancia Markatch Aike, fue identificado el Abrigo Pintado de Markatch Aike -APMA- además, de otras pinturas que se encontrarían muy próximas entre sí (Molina, 1972), y cuyas localizaciones son difíciles de precisar a partir de las descripciones proporcionadas. Asimismo, se señala la presencia de motivos que se distribuyen sobre la extensa colada basáltica conocida localmente como la "Muralla China" -MCh 1-8- (Hernández Llosas et al. 1999) (Fig. 1).

En ausencia de dataciones absolutas se acepta que las pinturas pueden corresponder a registros de ocupaciones tardías, ubicadas en torno a los últimos 2000 años AP (Bate, 1970, 1971).

A partir de la distribución de las pinturas rupestres en el CVPA se postularon diferencias en las clases de motivos y en la proporción en que 


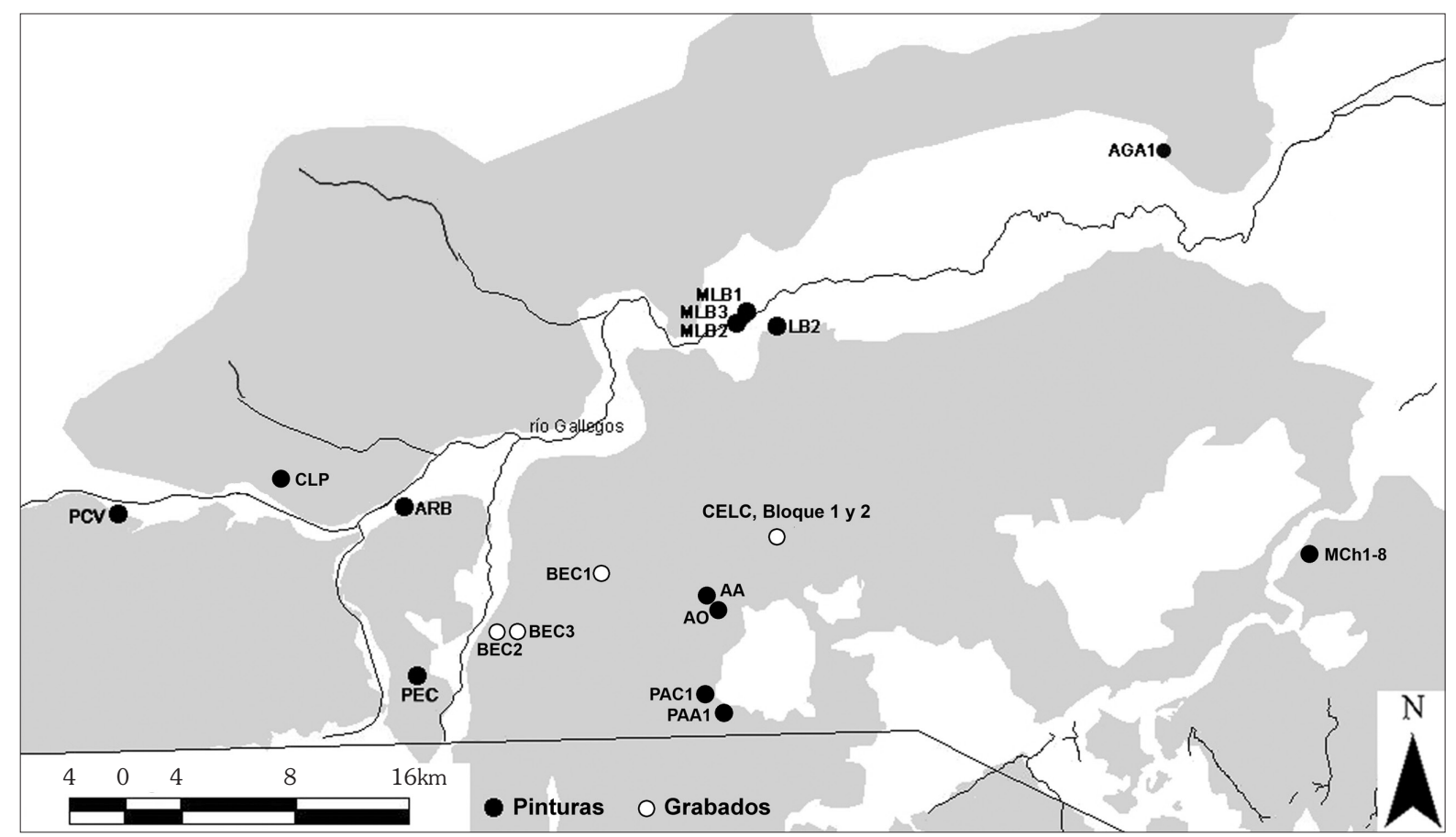

Fig. 1. Distribución localizaciones con manifestaciones rupestres "estilo río Chico" en la cuenca de los ríos Gallegos y Chico.

algunas de ellas están representadas en Última Esperanza -sector chileno- (Bate, 1970, 1971; Gallardo, 2009; Massone, 1982), en el río Gallegos y en la porción que el río Chico recorre el sector argentino (Charlin \& Borrero, 2012; Hernández Llosas et al. 1999; Gómez Otero, 1986-87; Manzi \& Carballo Marina, 2012). A esta variabilidad, ya reconocida en las manifestaciones rupestres de la cuenca del río Gallegos, se suman, en su sector medio, la presencia en cuatro localizaciones donde fue detectada la técnica de grabado en la manufactura de las mismas.

\section{LOS GRABADOS RUPESTRES}

Los grabados fueron identificados en las estancias Bella Vista y La Carlota, en ambos casos en relación con las laderas de coladas basálticas que enmarcan dos cuencas endorreicas (Fig. 1).

En primer lugar se describen los grabados relevados en la estancia Bella Vista, los que se ubican en una colada basáltica (200 msnm) que muestra laderas con pendientes fuertes (20\%) y desniveles que alcanzan los $50 \mathrm{~m}$. Están afectadas por proceso de remoción en masa, principalmente desmoronamientos de bloques de basalto (Mazzoni et al. 2001). Esta colada se vincula con una cuenca endorreica de grandes dimensiones y muy profunda en la que se detectaron diferentes líneas de costa que evidencian modificaciones en su tamaño. En las laderas se ubican afloramientos de agua que forman mallines con mayor cobertura vegetal y buena calidad forrajera (Mazzoni, 2001). Esta cuenca se alimenta de agua de lluvia por lo que presenta gran variabilidad interanual; fue vista seca en 1999 y en el año 2002, se la observó con siete m. de agua (E. Mazzoni, com. pers. 2015). Como se ha mencionado es conocida localmente como "Bajo El Cóndor" o "Bajo Bella Vista" y si bien se trata una única colada los soportes utilizados para plasmar los grabados no son los mismos. Sobre el borde oeste se localizaron una cueva de pequeñas dimensiones -denominada sitio Bajo El Cóndor 2-y una pared rocosa -sitio Bajo El Cóndor 3-. Fuera del bajo y al noroeste de las anteriores, se ubica otra cueva -sitio Bajo El Cóndor 1- con grabados y pinturas. Todos ellos se corresponden con los sectores más estables de la ladera (Mazzoni et al. 2001).

Por último, existen referencias acerca de 
una cueva localizada en el farallón norte de la colada basáltica, que se encuentra colmatada arena y presenta motivos pintados atribuibles al "estilo Río Chico" (J. B. Belardi, 2010, com. pers. y M. Vázquez, 2014 com. pers.). Distante unos 3,5 km aproximadamente y en sentido SE de este sitio, se registraron dos aleros que también presentan pinturas rupestres, denominados Alero de las Ortigas -AO- y Alero del Artista -AA-, separados entre sí por unos $400 \mathrm{~m}$.

Cabe señalar que se observaron artefactos líticos expuestos en los taludes de la colada basáltica, información que será dada a conocer en próximos trabajos.

\section{Bajo El Cóndor 1 -BEC1-}

Esta cueva, orientada hacia el S-SO, de 2,30 $\mathrm{m}$ de ancho, 1,80 $\mathrm{m}$ de alto y 1,50 $\mathrm{m}$ de profundidad presenta paredes laterales $e$ interior con evidencias de manifestaciones rupestres. Se registró un total de 25 motivos de los cuales diez son pinturas ejecutadas con tratamiento lineal en tonos rojo carmesí y otros 15 corresponden a grabados realizados mediante picado. No obstante, se observa un motivo (trazos) en el que se conjugan el picado y el abradido.

Los diseños grabados comprenden a: Arco simple (1), Círculos concéntricos (1), Círculos concéntricos con arcos en la porción superior (1), Indeterminados por morfología (2), Trazos horizontales paralelos con apéndices (1), Trazos compuestos oblicuos cruzados (1), Tridígitos simples (5) y Tridígitos compuestos (3). En tanto que los diseños de las pinturas corresponden a: Círculo simple (1), Indeterminado por morfología (1), Trazo en "V" simple (1), Trazos en "V" dobles (1), Trazo simple oblicuo (2), Trazos compuestos paralelos oblicuos (1), Trazo simple horizontal (1) y Tridígito (2) (Fig. 2).

Las pátinas de los motivos grabados y de los pintados parecen ser semejantes. No obstante, se destaca la presencia de un tridígito ubicado fuera del área reparada que tiene una pátina más clara que el resto de los motivos. No es fácil dirimir si corresponde a un episodio de ejecución más tardío o bien estaría afectado por su mayor exposición a los agentes atmosféricos. Asimismo, se registra un caso de superposición de motivos donde un

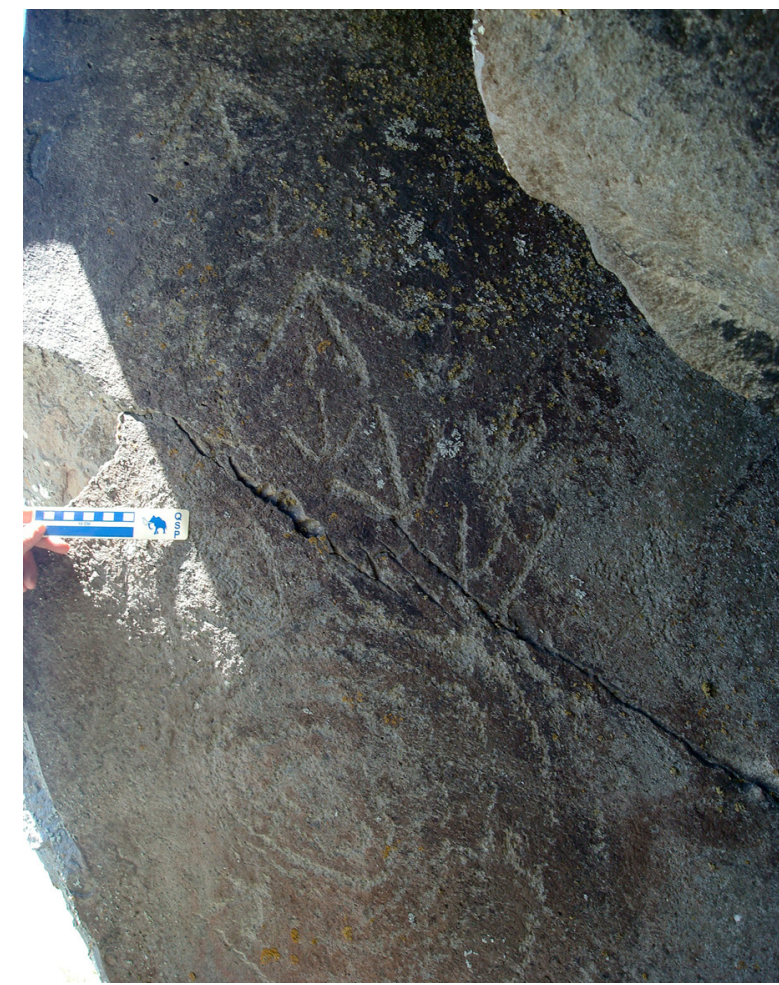

Fig. 2. Bajo El Cóndor 1 -BEC1-. Grabados rupestres: círculos radiados con apéndices y tridígitos.

arco grabado se ubica por debajo de un motivo pintado indeterminado por morfología, el que a su vez se encuentra por debajo de un tridígito simple grabado. Esto indicaría al menos tres momentos distintos en la ejecución de los mismos.

El estado de conservación de las representaciones es en general bueno, aunque se observan cinco motivos grabados que están colonizados por líquenes. En una unidad topográfica próxima a esta localización -ca. 200 m- se encuentra un conjunto de incisiones, al parecer subactuales, que no afectan directamente a los motivos antes mencionados. Se trata de incisiones finas que se encuentran patinadas y están conformadas por trazos largos verticales y oblicuos, un enrejillado y dos posibles letras del alfabeto latino -una ele (L) y una eme (M) mayúsculas, ambas en letra cursiva-.

\section{Bajo El Cóndor 2 -BEC 2-}

Sobre la pared oeste de la colada basáltica se localizó un reparo orientado al E-NE de 2,27 m de ancho, 2,47 $\mathrm{m}$ de alto y 1,15 $\mathrm{m}$ de profundidad. 
Hacia el frente y sobre el lado sur de la boca, se ubica un bloque caído que presenta una cara de $0,65 \mathrm{~m}$ de largo y $0,14 \mathrm{~m}$ de ancho sobre la que se disponen tridígitos. En el lado norte de la misma hay otro bloque de 0,44 m de largo x 0,35 m de ancho, que muestra un conjunto de incisiones finas que en algunas porciones presenta pulido, posiblemente subactual.

El área total sobre la que se disponen las manifestaciones rupestres, considerando el bloque con los tridígitos, la cueva y sus paredes laterales, es de cuatro metros lineales. Estas comprenden grabados ejecutados mediante técnicas de abradido y picado, como así también pinturas con tratamiento lineal en tono carmesí.

Los motivos grabados ascienden a cinco y el pintado a uno. Los diseños grabados comprenden Tridígitos (1), Circunferencias (1), Óvalo (1) e Incisiones finas (1). Mientras que el pintado es una Mancha (1). Algunos de estos motivos grabados (circunferencias, óvalo e incisiones) junto con las múltiples incisiones finas -como las observadas en una pared cercana- presentan pátinas atribuibles a una manufactura más reciente. No obstante, en el caso de las circunferencias, la casi ausencia de pátina podría deberse, tal vez, a que se encuentran protegidas en el interior del reparo (Figs. 3a y 3b).

\section{Bajo El Cóndor 3 -BEC3-}

En esta pared rocosa los motivos rupestres se distribuyen en una unidad topográfica de 1,34 m de ancho por 1,08 $\mathrm{m}$ de alto, con orientación E-ES.

Se registró un total de 20 motivos ejecutados

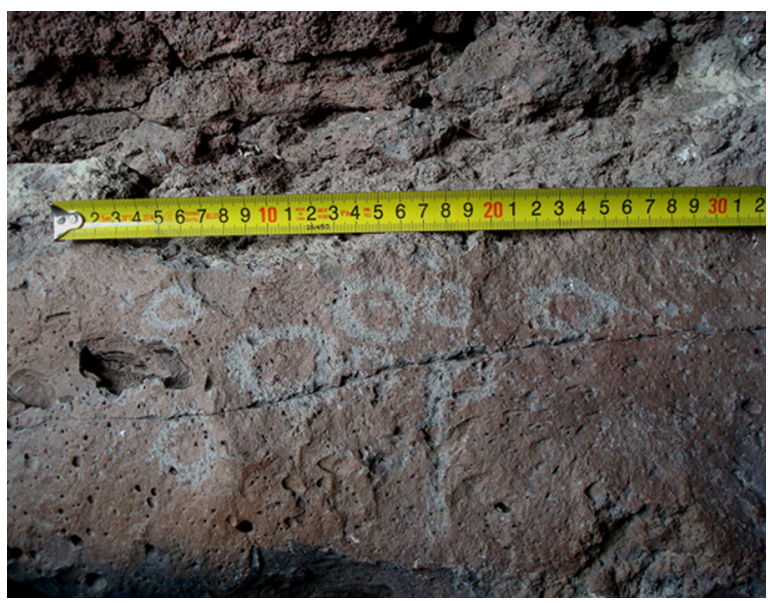

Fig. 3a. Bajo El Cóndor 2 -BEC2-. Circunferencias. mediante grabado haciendo uso de las técnicas de abradido (13), picado (3), inciso (2) e incisopulido (2). Los diseños representados comprenden Motivos en "U" (1), Indeterminados por morfología (2) y Tridígitos (18). Estos últimos tienen la particularidad de no evidenciar vínculos asociativos por lo que fueron categorizados como motivos simples (pisada). Sólo en un caso se identificó un motivo compuesto por dos elementos, dando idea de rastro. En otro, los dedos externos en lugar de tener forma de "V", como en la mayoría de los diseños, tienen forma de "U".

En esta unidad topográfica se constata un caso de superposición entre motivos. Se trata de dos pisadas donde una -ejecutada mediante abradido- se encuentra por debajo de otra incisa y pulida. Los diferentes grados de pátina reconocidos permiten diferenciar un conjunto de motivos más recientes que corresponden a diseños producidos mediante las técnicas de picado, inciso e inciso y pulido (Fig. 4).

El cuarto lugar donde se detectaron grabados es en la Sección Cerro Negro de la estancia La Carlota. Se localizan a unos $14 \mathrm{~km}$ al noreste del

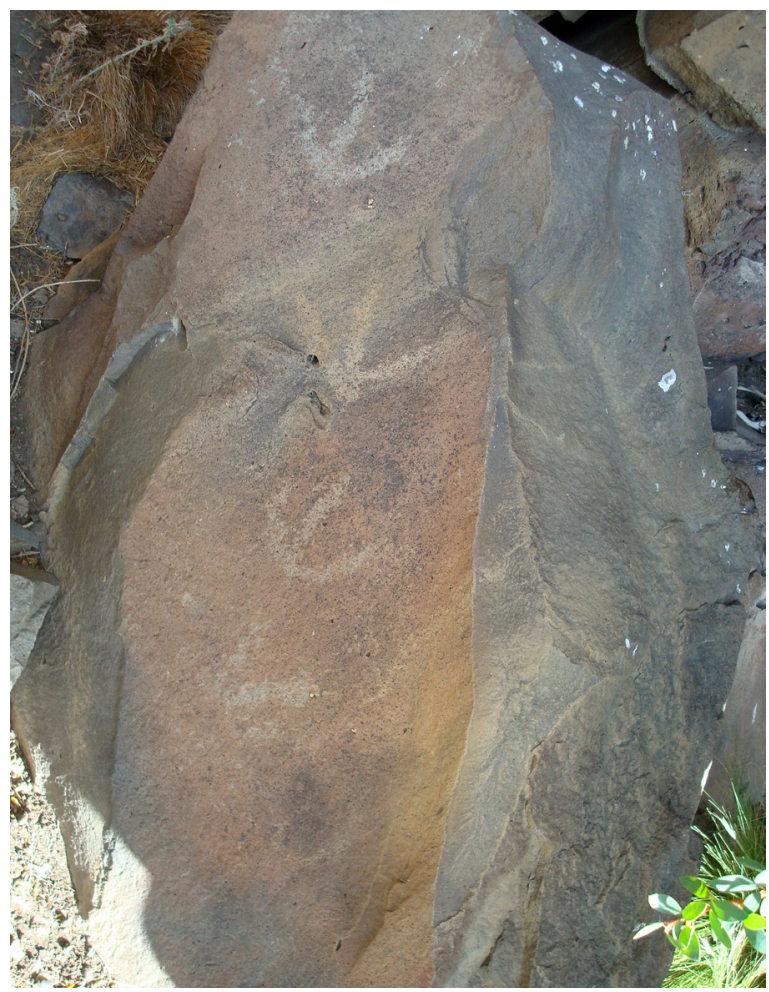

Fig. 3b. Bajo El Cóndor 2 -BEC2-.Tridígitos. 


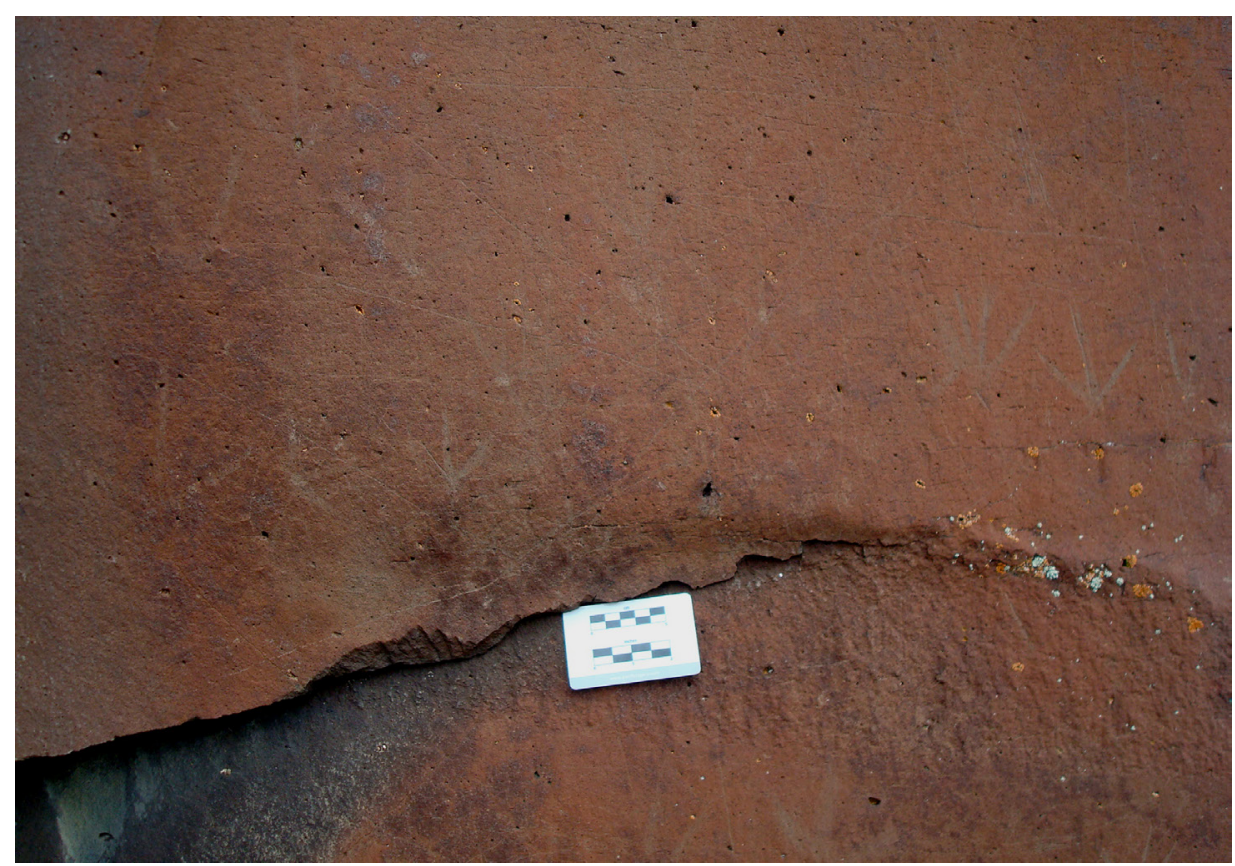

Fig. 4. Bajo El Cóndor 3 -BEC3-. Panel con grabados.

Bajo El Cóndor y unos $6 \mathrm{~km}$ en igual orientación de la laguna Potrok Aike (Fig. 1). En esta ocasión, se trata de una colada basáltica (150 msnm) muy fragmentada, relacionada con una cuenca endorreica cuyo borde este expone rocas de grano fino de muy buena calidad para la talla. Es de destacar que en la misma ladera, con acumulaciones de arena intercaladas con bloques de basalto desprendidos de la colada, se identificó en superficie alta frecuencia de artefactos líticos confeccionados en basalto de muy buena calidad para la talla.

Sobre la ladera sureste en dos bloques de basalto, distantes unos $5 \mathrm{~m}$ entre sí, se reconoció grabados. Fueron denominados Campo El Cinco, Bloques 1 y 2 (CELC, Bloques 1 y 2). En el Bloque 1 , de mayores dimensiones, los grabados se presentan agrupados en torno a unos 0,60 $\mathrm{m}$ de largo y $0,40 \mathrm{~m}$ de ancho. Se identificaron nueve motivos ejecutados mediante abradido, entre los que se distinguen: Trazos paralelos con uno cruzado -en $\mathrm{H}$ - por el medio (1), Tridígito (1), Indeterminados por morfología (5), Arco (1) y Rectángulo con diseño interno (1). Estos dos últimos presentan una pátina más oscura que los restantes pudiendo ser parte de un diseño compuesto por dos elementos o marcar episodios distintos de ejecución (Fig. 5).
En el Bloque 2 los motivos identificados fueron ejecutados mediante la técnica del abradido. Se concentran en una superficie de $0,40 \mathrm{~m}$ de largo x 0,40 m de ancho y comprenden: Círculo (1), Círculo con apéndice (1), Arco con diseño interno (1), Trazo vertical (1) e Indeterminado por morfología (1). Se observan dos casos de superposiciones, Círculo/Arco con diseño interno y Trazo vertical/Indeterminado por morfología (Fig. 6). Las pátinas en este bloque serían semejantes $e$ incluso podría proponerse una correspondencia con las más claras del Bloque 1.

\section{Consideraciones finales}

Se destaca que los sitios con grabados rupestres corresponden al primer registro de este tipo de manifestación visual en la cuenca del río Gallegos. Quedan comprendidos entre los diseños atribuibles al "estilo Río Chico" propuesto a partir de pinturas rupestres registradas en el extremo sur de Patagonia. Entre ellos, se constatan los motivos de trazos, círculos, arcos, motivos en "U" y tridígitos, como los más frecuentes.

En general, puede sostenerse que las clases de motivos englobados en aquel estilo presentan una alta simplicidad de diseño que favorecería su 


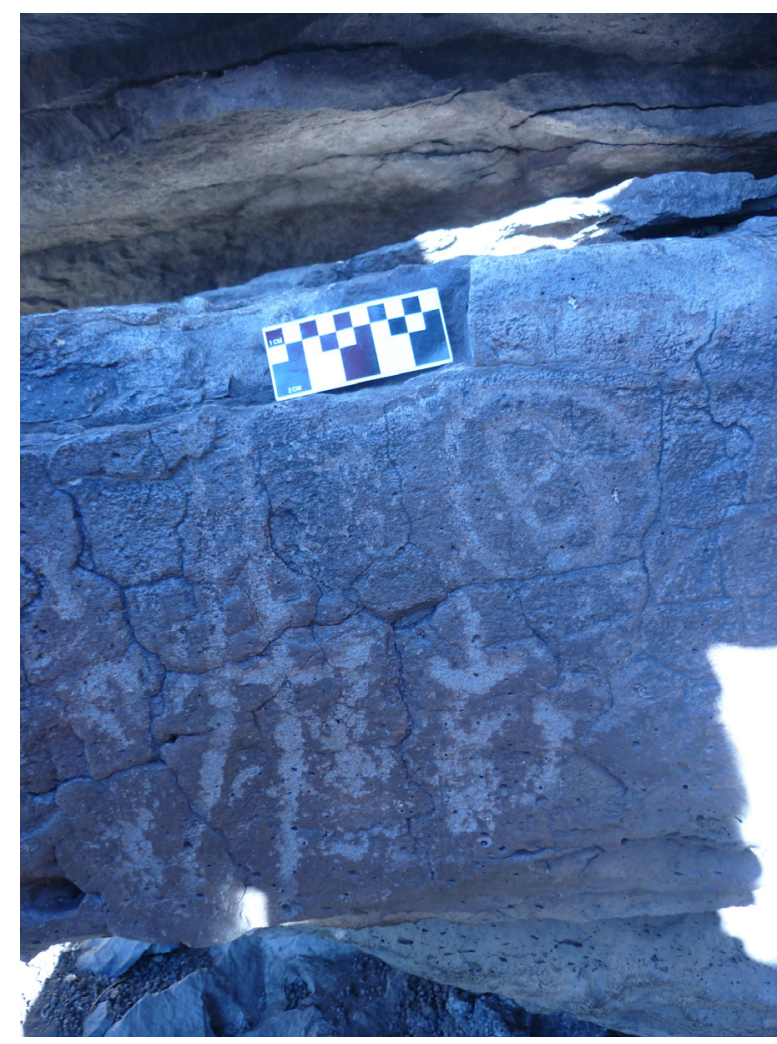

Fig. 5. Campo El Cinco, Bloque 1. Grabados. réplica. Mientras que los figurativos, en este caso únicamente tridígitos, presentan una distribución megarregional, lo que también los torna fáciles de identificar en diversas localizaciones. En síntesis, se enfatiza que es el proceso técnico de producción de los grabados lo que los hace diferentes a las pinturas.

A pesar de lo intensivo de las prospecciones, la alta disponibilidad de soportes y la frecuencia de pinturas, en el sector septentrional del CVPA se observan grabados sólo en cuatro sitios arqueológicos de la cuenca media del río Gallegos. Los más extremos -CELC Bloques 1 y 2 - y BEC2 y 3 se encuentran distantes entre sí por unos 14 $\mathrm{km}$ lineales y se hallan circunscriptos a un cuerpo de agua de régimen temporal -Bajo El Cóndor- y a otro permanente -laguna Potrok Aike-.

La coocurrencia de pinturas y grabados se vincularía tanto con la presencia de agua permanente, la existencia de mallines localizados en las laderas de las coladas con agua y pasturas y la evidencia de abrigos rocosos con ocupaciones intensas y redundantes -Potrok Aike, cueva 1 (Gómez Otero, 1986-1987) y La Carlota, cueva 1 (Campan et al. 2007). Por último, cabe mencionar la alta disponibilidad y accesibilidad de rocas de muy buena calidad para la talla y en alta frecuencia como el basalto en la laguna Potrok Aike

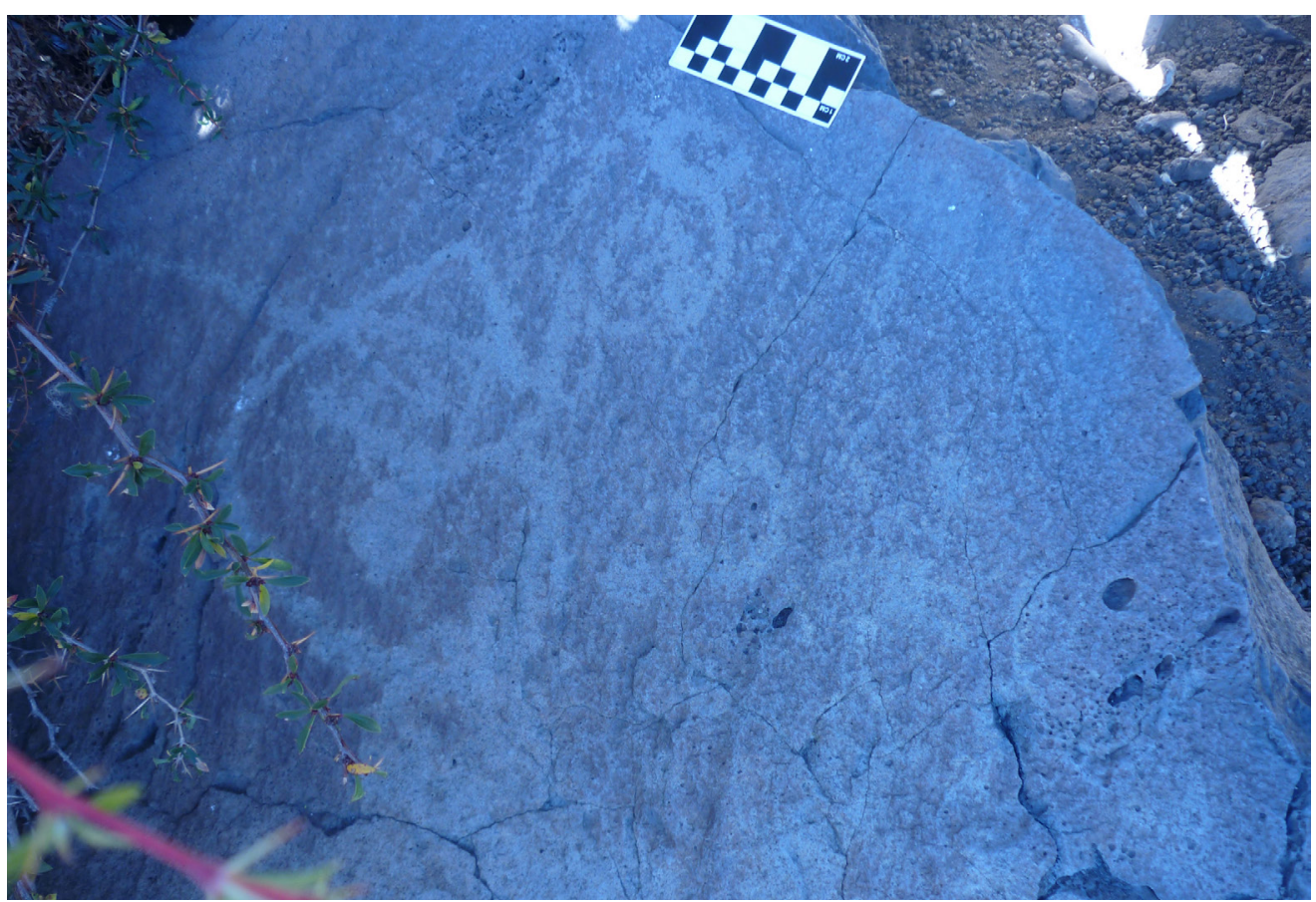

Fig. 6. Campo El Cinco, Bloque 2. Grabados. 
(Charlin, 2009) y la calcedonia en las laderas de la meseta La Carlota (Campan et al. 2007).

\section{Agradecimientos}

$\mathrm{Al}$ administrador de la estancia Bella Vista Sara Braun S.A. Brian Olaf Halborsen, por facilitarnos las tareas de campo. A Romario Barría, por informarnos de los grabados en la estancia La Carlota. A Sabrina Leonardt, por las fotos. A Mirian Vázquez por la información recibida y a Juan Bautista Belardi por la lectura del manuscrito. Este trabajo se inscribe en el PIP-CONICET 1122009010020101.

\section{BIBLIOGRAFÍA}

Bate, L. (1970). Primeras investigaciones sobre el arte rupestre de la Patagonia chilena. Anales del Instituto de la Patagonia, 1(1), 15-26.

Bate, L. (1971). Primeras investigaciones sobre el arte rupestre de la Patagonia chilena (segundo informe). Anales del Instituto de la Patagonia, 2, 33-41.

Campan, P., Carballo Marina, F., \& Manzi, L. (2007). Arqueología de Estancia La Carlota (Campo Volcánico Pali Aike, Argentina). En F. Morello, M. Martinic, A. Prieto, \& G. Bahamonde (Eds.), Arqueología de Fuego- Patagonia. Levantando piedras, desenterrando huesos... y develando arcanos (pp. 687-700). Punta Arenas: CEQUA.

Charlin, J. (2009). Estrategias de aprovisionamiento y utilización de las materias primas líticas en el campo volcánico Pali Aike (Prov. Santa Cruz, Argentina). BAR International Series, No. 1901, Archaeopress, Oxford.

Charlin, J., \& Borrero, L. A. (2012). RockArt, Inherited Landscapes, and Human Populations in Southern Patagonia. En J. Mc Donald \& P. Veth (Eds.), A Companion to Rock Art (pp. 381-397). Oxford: Blackwell Publishing Ltd.

Charlin, J. (2014). Nuevos sitios con representaciones rupestres en la localidad de Potrok Aike (Santa Cruz, Argentina). Magallania, 42(1), 187-200.

D' Orazio, M., Agostini, F. Mazzarini, P., Innocenti, M., Manetti, M., Haller J., \& Lahsen, A. (2000). The Pali Aike Volcanica Field, Patagonia: Slab-Window Magmatism Near The Tip of South America. Tectonophysics, 321, 407-427.

Gallardo, F. (2009). Sobre la composición y la disposición en el arte rupestre de Chile: consideraciones metodológicas e interpretativas. Magallania, 37(1), 85-98.
Gómez Otero, J. (1983-1985). Investigaciones arqueológicas en las cuencas media y superior del río Gallegos. Informe Final Beca de Perfeccionamiento CONICET. Buenos Aires. MS

Gómez Otero, J. (1986-1987). Investigaciones arqueológicas en el alero Potrok-Aike (Provincia de Santa Cruz). Relaciones, XVII (I), 173-198.

Gómez Otero, J. (1988). Investigaciones binacionales en la zona fronteriza argentino-chilena (paralelo $52^{\circ} \mathrm{S}$ ), entre el valle del Río Chico y del Gallegos Chico. Informe CONICET. Buenos Aires. MS.

Gómez Otero, J. (1989-1990). Cazadores tardíos en la zona fronteriza del paralelo $52^{\circ}$ sur. El paraje de Juni Aike. Anales del Instituto de la Patagonia, 19, 47-71.

Hernández Llosas, M. I., Nami, H., \& Cuadrado Woroszylo, M. (1999). Arqueología en la localidad arqueológica de Pali Aike, cuenca del río Chico. II. Resultados preliminares sobre las representaciones rupestres. Praehistoria, 3, 202-217.

Maerz, A. J., \& Paul, M. R. (1930). A Dictionary of Color. Nueva York: McGraw-Hill.

Manzi, L., Carballo Marina, F., \& Campan. P. (2010). Manifestaciones rupestres y distribuciones artefactuales en el campo volcánico Pali Aike (sector medio del río Gallegos, Santa Cruz): nuevas evidencias arqueológicas. Actas del XVII Congreso Nacional de Arqueología Argentina, (pp. 80-86). Mendoza.

Manzi, L., \& Carballo Marina, F. (2012). Manifestaciones rupestres en el campo volcánico Pali Aike (cuenca del río Gallegos, Santa Cruz, Argentina). Magallania, 40(1), 283-302.

Massone, M. (1982). Nuevas investigaciones sobre arte rupestre de Patagonia meridional chilena. Anales del Instituto de la Patagonia, 13, 73-94.

Mazzoni, E. (2001). Distribución espacial y caracterización geomorfológica de "bajos sin salida" de la Patagonia Austral Extracordillerana. Anales del Instituto de la Patagonia. Serie Ciencias Naturales, 29, 5-24.

Mazzoni, E., Ruiz Posse, E., Vazquez, M., Baetti, C., \& Martínez, G. (2001). Unidades de paisaje en pequeñas cuencas endorreicas de la Patagonia Extracordillerana Argentina. Anales de la Sociedad Geográfica Chilena de Ciencias Geográficas, 95-102.

Molina, M. (1972). Nuevos aportes para el estudio del arte rupestre patagónico. Anales de la Universidad de la Patagonia "San Juan Bosco", 4, 64-182.

Sanguinetti de Bórmida, A. (1981). Introducción a la prehistoria de la Patagonia, Argentina. Tesis doctoral. Facultad de Filosofía y Letras, Universidad de Buenos Aires. Buenos Aires. MS. 